Boris Zhelenkov - Irina Safonova - Yakov Goldovsky - Elizaveta I. Dmitrieva

\title{
FORMATION AND OPTIMIZATION OF TRANSPORT NETWORKS IN THE CONDITIONS OF THE DIGITAL INTEGRAL ENVIRONMENT FOR TRANSPORTATION
}

The article provides a brief description of the digital integrated transportation support environment. The methods of choosing the base route in the formation of transport networks in the conditions of the formation of a digital integrated environment are presented. It is shown that the means of solving the problem of optimization of transport networks is a developed modeling complex, which consists of a set of models with the necessary degree of detail. The paper presents a functional diagram and a brief description of the complex. The complex functions taking into account the replenishment, improvement and updating of models, allow you to combine different types of models and use the necessary of them when solving specific problems of transport network optimization. The modeling complex allows to significantly simplify the optimization of transportation networks. On the basis of the proposed mathematical apparatus, the software of an automated system has been developed, which is designed to function in a digital integrated environment, a brief theoretical description of which is given.

Keywords: digital integrated environment, basic route, route criteria and parameters, transport network, optimization, models, modeling complex

\section{Introduction}

The problem is the organization of the information space in which both carriers and customers would feel comfortable is very urgent now. To solve this problem, it is necessary to create a digital integrated environment - which is the main component of a unified information space to meet the needs of the transportation of passengers and goods, where user work is positioned as an electronic service. The digital integrated environment should have a modular structure that will enable the connection of new routes, new carriers, as well as representatives of related organizations to provide additional services. When creating new routes, it is necessary to evaluate the parameters of the routes and optimize the movement of route vehicles. Consequently, an important step in the development of a digital integrated environment for transportation is the optimization of route transportation networks.

The digital integrated environment for transportation is a single information space implemented by software and hardware, which provides the opportunity for interaction between carriers and consumers. The digital integrated transport environment is designed to provide information on all types of transport networks, including urban, regional, intercity, personal and freight transport

The tasks of modeling and optimizing route transportation networks were taken up by many specialists. A great contribution to the mathematical formulation of optimization problems and the development of models of the movement of route vehicles made Varelopulo,
Lieberman, Arak, Ligum, Levinson, Black, Vuchic, Schnabel, Lohse, Fernandez and other scientists.

Today there are many mathematical models of optimization of transport networks [1-2]. However, all these models are separated and presented in different ways, have a different form of recording, different representation of input and output parameters, the same parameters in different models have different units of measurement, etc. At the moment there is no single unified form, a single method of representation of such models, while individual models do not allow to fully solve the necessary optimization problems in a digital integrated environment. Therefore, the development of new more effective models, approaches and software to optimize transport networks applicable in the digital integrated environment is an urgent scientific problem.

\section{Transport network design and optimization}

A single information space for transportation is a space for interaction between participants in the transportation process: employees of transport companies, customers, authorities and other parties interested in the quality of transportation. A single information space for transport provision arises as a result of the formation of an appropriate digital integrated environment, including [3-4]:

- $\quad$ automated control systems of transport companies;

- clients of transport companies and authorities;

- governmental institutions;

Boris Zhelenkov ${ }^{1}$, Irina Safonova ${ }^{1, *}$, Yakov Goldovsky ${ }^{1}$, Elizaveta I. Dmitrieva ${ }^{2}$

${ }^{1}$ Department "Computing systems and networks" of the Russian University of Transport (RUT - MIIT), Russia

${ }^{2}$ Department "Foreign Languages 5" of the Russian University of Transport (RUT - MIIT), Russia

*E-mail of corresponding author: iesafonova@yandex.ru 


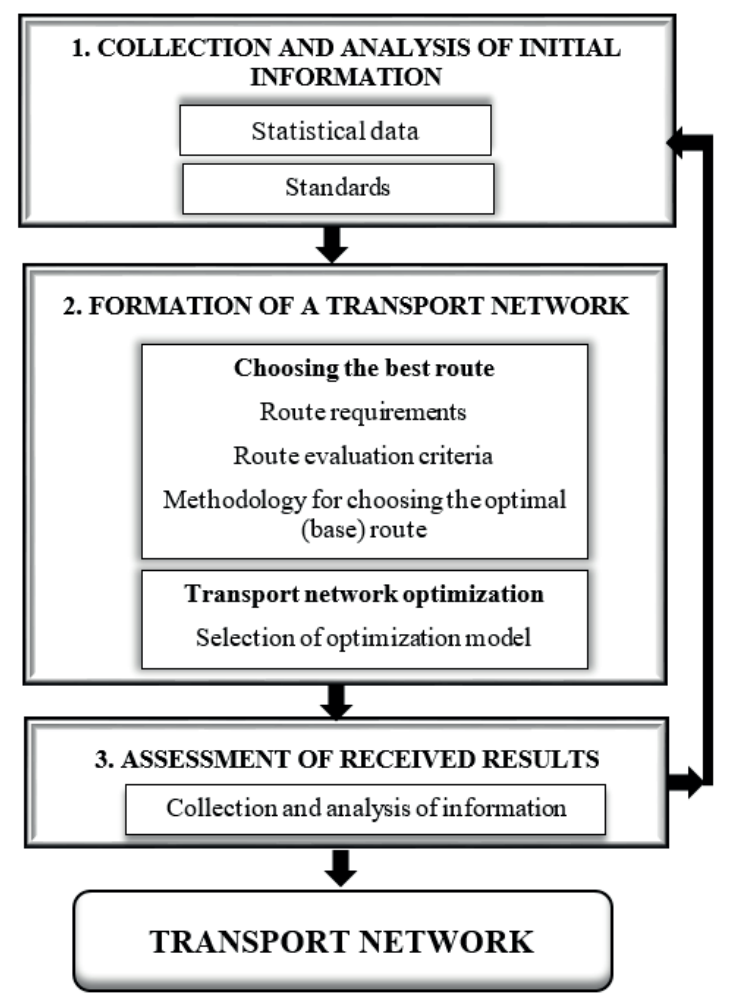

Figure 1 Scheme for transport networks design

- corporate and local telecommunication networks, expert systems, technological databases and reference books;

- regulatory databases;

- $\quad$ specialized data processing centers that provide timely processing of large volumes of information;

- on-board devices and mobile devices that collect data directly during transportation;

- digital communications in the form of global telecommunication networks, connecting all the listed components into the information environment.

However, the digital integrated environment is not yet a single information space. A single information space, in addition to solving other important tasks, requires the development of standards and protocols that ensure a wide exchange of information between its components. To do this, the digital integrated environment must be provided for joint work and efficient data exchange between:

- $\quad$ automated transport management systems;

- digital interfaces of clients of transport companies;

- a single portal of public services for the access of employees and customers of transport companies to information;

- geographic information systems, including electronic maps, 3D-terrain models and decrypted satellite photos;

- a unified system of identification, authentication and authorization of users of the digital platform;

- payment service to pay for transportation, mutual settlements of participants in the transportation process, payment of taxes and fees;
- a system of reference books, classifiers, registers and other regulatory reference information.

The concept of using such a space involves registering a participant in the transportation process on the service portal, either as a client or as a representative of the carrier. The client should be given the opportunity to choose transportation services based on their preferences. For example, if cargo is transported, the determining factors should be the parameters of the cargo, as well as the customer's preferences - price, speed of delivery, etc. Based on these and other input data, the route is built. On the way of the cargo, the points of trans-shipment are determined, and the client receives information about the route and the final cost of the service. When implementing passenger transportation, it is necessary to take into account not only price and time parameters, client preferences for choosing possible intermediate points (cities, attractions), with an opportunity of creating a route map with the necessary transport documents, up to booking hotel rooms and excursions.

To implement a digital integrated environment, it is necessary to solve a number of problems: development of a methodology for creating a digital integrated environment for transport; development of standardization methodology in the field of digital transportation management; development of a set of models for the implementation of a digital integrated environment; development of the concept of connecting carrier modules; development of an intelligent interactive interface with a digital integrated environment (carrier/customer). In addition to the above, an 
important task in creating a digital integrated environment for providing transportation is the task of forming and optimizing transportation networks. A transport network is a set of possible routes of all modes of transport included in the process of transporting passengers or goods between two endpoints. Since several modes of transport can be used for one route, such a system can be called an integrated transport infrastructure or environment. The process of creating transport networks is presented as a diagram in Figure 1.

The process of design of transportation networks includes three main stages:

Stage 1. Collection and analysis of initial information. Information is collected from video monitoring systems and DVR systems. They also include video monitoring of public transport and outdoor security cameras. To collect and analyze initial information, the following are needed: systematization and standardization of video recording systems; systematization of information processing. It is necessary for the control system to include the means of satellite navigation.

Stage 2. Formation of the transport network.

\subsection{Choosing the best route for inclusion in the transport network}

At this stage it is necessary to make an informed decision about which route should really be included in the transport network, using the method of choosing the optimal route (it is reasonable to call it a base route) from a variety of alternatives.

Generally, when there is an ambiguity in the choice of a route (usually this occurs under conditions of uncertainty in the initial information), i.e. the only best option cannot be found. You can get only a few («area») rational options. Considering this circumstance, one should first identify this set of rational routes, the final choice of the optimal (base) route that will be included in the transport network is made by applying various expert procedures. With such a final choice, certain formalized techniques can be applied (for example, expert assessment methods, hierarchy analysis method), however, the main role belongs to the person - the expert.

\subsection{Optimization of transport networks}

The study of modern methods for optimizing transport networks has shown that since the beginning of the 2000s, new approaches to building and optimizing transport networks have been actively introduced in European countries [5]. Existing optimization models should be considered here and/or completely new ones should be developed.

Stage 3. Evaluation of the results obtained is also carried out by collecting information from video monitoring systems, from entrance and exit turnstiles, etc. Analysis of video processing is carried out using innovative technologies in the field of artificial intelligence. When analyzing the video sequence, additional opportunities arise for the control of emergency situations. The processing of information received from video monitoring systems can be performed locally or centrally. Local processing of information requires the presence of an on-board specialized computer on a vehicle or traffic control means (traffic lights). The result will be transferred to the dispatch center. Centralized processing of information reduces the requirements for local computing facilities, but increases the load on data transmission channels. At this stage, a set of measures to ensure reliability and information security is being developed.

The result is a transport network - an electronic map with complete information about the state of traffic flows, emergency situations and the loading of vehicles on routes.

\section{Choosing the optimal route for the transport network}

Passenger or cargo can be moved between two endpoints using several modes of transport. Each type of transport has its own network of routes that are already formed and regularly used in various geographical areas. When you include any type of transport in the integrated transportation system, all its routes in the corresponding geographical territory are added to the database and can be used to build the necessary integral (composite) route. By integral route we mean a route using various modes of transport. When creating a digital integrated transport support environment, existing routes can be completely transferred to the digital integrated environment, and new routes will be added taking into account the existing ones.

To determine new routes, it is necessary to evaluate the parameters of routes and optimize transport networks. Estimation of route parameters and optimization of transport networks leads to increased profitability and quality of services of transport companies.

When choosing the best route one should use an appropriate method of selecting the best option from a variety of alternatives. The choice of a route is a multicriteria task, and many of these criteria are difficult to describe accurately. It is necessary to determine and take into account all the requirements for the route, parameters and criteria for its evaluation. Route parameters are - mode of transport, traffic fluctuations, peak loads, risks, cargo parameters, infrastructure condition, range, time delays of the route, tax rates, volume and condition of the fleet of transport companies, quality of delivery and many others.

Under the conditions of the formation of a digital integrated environment, when choosing a base route to form a transport network, it is advisable to use interactive decision-making methods, since this allows using the creative capabilities of a person - decision-maker (DM) [6-7]. An expert acts as a decision maker, for example, he can be a transport company manager, government official, 
etc. However, in the process of developing the digital integral medium of transportation, there will be a tendency to unite the means of neural networks, expert systems in creating multi-stage decision support systems for the formation of route networks.

The choice of the base route should be made taking into account all the factors that determine the quality of such a decision, including technical and organizational, as well as cost, and time ones. This requires the use of specific techniques and methods involving the use of intuition and the decision-maker's experience. Decisions made must be steady and regular. Stability of solutions should be understood as the property to remain unchanged with respect to changes in a priori probabilities affecting the values of the estimated functionals. Decision stability analysis allows DM to identify solutions with a certain guaranteed level. A stable solution is different in that it is resistant to DM errors in terms of a priori probabilities. Regularity of decisions means that a complete regular solution has the property that it is optimal for all criteria considered, but this is extremely rare. In this case, it is advisable to use the set with partial regularity of the solution, which is optimal only for individual criteria.

In formal terms, the following decision-making conditions that arise when choosing a base route during the formation of a transportation network [8-9] can be distinguished: certain conditions when all the information for forming a base route is precisely known; conditions of uncertainty, when, in addition to unambiguous source data, there are random variables with precisely known probabilistic characteristics, as well as quantities for which their probabilistic description is not precisely known or not known at all.

A study of decision making methods showed that for conditions of certainty of initial information about routes, it is advisable to use the lexicographic method of organizing a variety of alternative options when choosing a base route [10]. The essence of the method is to streamline the decisions in order of criteria importance. In the process of choosing a base route from the set of route parameters, you should choose the one that, in the specific application conditions, reflects the necessary route properties, for example, time delays, and gives it a criterion function.

As practice shows, the majority of decision-making tasks on choosing a route to include it in the transport network are solved under conditions of uncertainty of the initial information. Analysis of existing approaches to solving the problem of choosing the base route in the face of uncertainty of the initial information showed the feasibility of a two-stage decision-making process. At the first stage, a set of rational routes is determined on the basis of formal methods, on the second, a basic route is adopted on the basis of expert procedures. Effective ones are the methods of analysis of payment matrices with the allocation of the main criterion and the method of analysis of hierarchies, respectively [11-12].

For the conditions of uncertainty of the initial information, the general algorithm for choosing the base route to be included in the transport network can be represented as follows:

A. Data input.

B. Criteria analysis.

C. The definition of a set of rational routes based on the method of analysis of payment matrices with the selection of the main criterion.

C.1 Selection of representative combinations of source data.

C.2 Search and preliminary analysis of solutions.

C.3 Calculation of the payment matrix.

C.4 Analysis of the payment matrix and the choice of rational decisions.

D. Selecting the base route using the hierarchy analysis method.

D.1 Assessing the importance of criteria by pairwise comparisons.

D.2 Calculation of criteria weights.

D.3 Comparison of the importance of alternatives by criteria.

D.4 Calculate the weights of alternatives for each criterion.

E. The end of the algorithm.

For the analysis of routes, the selection criteria proposed for the conditions of uncertainty are used - Wald, Laplace, Savage, Hurwitz criteria or their combination [13].

\section{Optimization of transport networks based on the modeling complex}

When modeling transportation in Russia, the software packages PTV Vision, AIM Sun and others are mainly used. Their main disadvantage is the closed architecture, that is, the impossibility of adding fundamentally new designs (calculation models). Existing modeling tools use only separate models and methods that do not allow to fully and adequately solve the tasks set for transport workers. A means of solving the task of optimizing transport networks is a modeling complex, which consists of many models that have the necessary degree of detail:

$M=\left\{M_{1}, \ldots, M_{i}, \ldots, M_{s}\right\}$

with $M_{1}, M_{i}, M_{s}$ being specific models.

The complex can include both analytical and simulation models for optimizing route networks. The functional diagram of the modeling complex is presented in Figure 2.

The fundamental properties of the modeling complex: property of integration, that is, the complex must integrate models depending on the specific situation (task);

- the property of evolution, which consists in the fact that the modeling complex, in fact, should be a working space for the gradual improvement of the transport network;

the property of duality, reflecting the possibility of representing the complex, on the one hand, as a model 


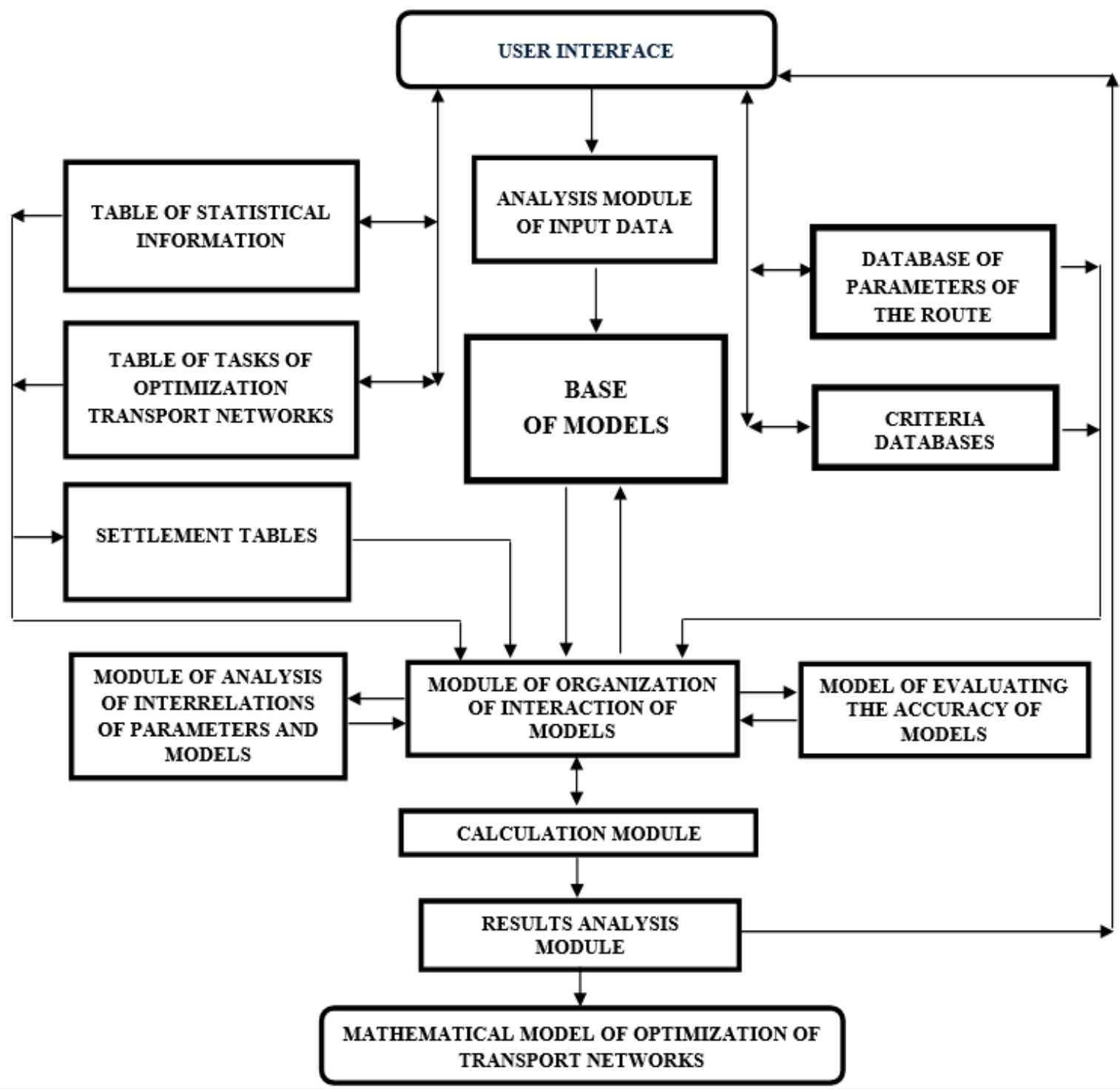

Figure 2 Functional diagram of the modeling complex

of the object under study, and, on the other hand, as a model, reflecting the process itself of optimizing the transport network;

versatility or applicability for solving an unlimited set of various problems of optimizing transport networks, taking into account the necessary parameters, criteria and restrictions;

the property of development, which consists in the fact that the complex is created and operates taking into account the replenishment, improvement and updating of optimization problems and models of their solutions; the complex allows you to combine different types of models and use the necessary ones in solving specific problems of optimization of transport networks in a digital integrated environment of transport.

Requirements for the modeling complex: adequacy; the possibility of refining the model when developing the transport network and the possibility of multi-level modeling; correctness of work; the ability to process data of large dimensions; effective software implementation; reliability; convenient visual perception.

In order to overcome the current diversity of the representation of the existing models for optimizing transportation networks, a single form and a unified methodology for presenting models, their input and output parameters, and units of measurement of parameters have been developed for the modeling complex.

The models of the complex should be presented in the form of the following dependence:

model $_{\text {model number }}:\{$ input parameters $\} \Rightarrow\{$ output parameters $\}(2)$

or , $M_{i}: \mathrm{X}_{\mathrm{pri}}^{\mathrm{in}} \Rightarrow \mathrm{X}_{\mathrm{pri}}^{\text {out }}$

with $\mathbf{X}_{\text {pri }}^{\text {in }}=\left\{x_{p r i 1}^{\text {in }}, \ldots, x_{\text {prin }}^{\text {in }}\right\}$ being input parameters for the $M_{i}$ model,

$\mathbf{X}_{\text {pri }}^{\text {out }}=\left\{x_{\text {pri }}^{\text {out }}, \ldots, x_{\text {pril }}^{\text {out }}\right\} \quad$ being $\quad$ output parameters (parameter) $M_{i}$. 
Taking into account Equations (2) and (3), Equation (1) is represented as a system of models as follows:

$$
M=\left\{\begin{array}{l}
M_{1}:\left\{x_{p r 11}^{i n}, x_{p r 12}^{i n}, \ldots, x_{p r 1 f}^{i n}\right\} \Rightarrow\left\{x_{p r 1}^{o u t}, \ldots\right\} ; \\
\vdots \\
M_{i}:\left\{x_{p r 1}^{i n}, x_{i 2}^{i n}, \ldots, x_{p r i j}^{i n}\right\} \Rightarrow\left\{x_{p r i}^{\text {out }}, \ldots\right\} ; \\
\vdots \\
M_{s}:\left\{x_{p r s 1}^{\text {in }}, x_{p r s 2}^{\text {in }}, \ldots, x_{p r s n}^{\text {in }}\right\} \Rightarrow\left\{x_{p r s}^{\text {out }}, \ldots\right\} .
\end{array}\right\},
$$

The output parameters of one model / models can be input parameters for another or other models. The number and types of models are determined by the nature of the problems of optimization of route transport networks.

It must be emphasized that Equations (2) and (3) determine only the form of representation of models, i.e. how various models for calculation should be presented in the base of complex models. This is necessary to simplify the work with various models of the complex, and to implement the fundamental properties of the modeling complex.

The composition of the modeling complex. Interface of user allows for problem-oriented communication between the user and the system that implements the operation of the complex.

In the databases (DB), the models and parameters of the models are presented, as well as the optimization criteria for transport networks. The tables contain a list of specific optimization problems and statistical information.

The module analysis of input data. The functional purpose of the module is to form a data structure. Data entry and selection of the optimization task is carried out at the user's request, while reading information from the corresponding tables and the database. The composition of the input data used may vary from one task to another.

The module of the organization of the interaction of models. Here processes of the organization of interaction of models are realized. On the basis of existing tables and databases, statistical processing of information about objects takes place - reduction of the dimension of the feature space, i.e. the selection of such a subsystem of features that would be smaller in volume than the prior space of features, and provided an acceptable solution. The analysis of the characteristics of models, analysis and coordination of units of measurement of input and output parameters of models in the process of solving each specific problem of optimization of transport networks, for example, the problem of calculating the coefficient of imposing passenger traffic on the transport network [14-15].

Module for analysis of relation between parameters and models. Functional purpose - determination of the necessary parameters to solve specific problems and the construction of criteria dependence.

Calculation module. The values of parameters obtained on the basis of theoretical calculation methods (by criteria) can be the initial data for subsequent calculations when optimizing transportation networks.

Module for assessing the accuracy of models. It is intended to assess the accuracy of each model considered when solving a specific task, to establish a quantitative relation between the input and output parameters of the models. The module implements the preparation and analysis of model data.

Results analysis module. According to the results of the analysis of the data obtained during the operation of the modeling complex, a message is displayed containing an assessment of the results obtained and, if necessary, recommendations for correcting them or adjusting the initial task of optimizing route networks.

The modeling complex functions as follows. Based on the data entered by the user (this may be an optimization problem, a set of route parameters, conditions and optimization criteria), the input information is analyzed. Next, we determine the necessary optimization tasks for transportation networks (available in the task table or completely new), the parameters to be obtained; necessary for solving selected problems of the model from the database. It is checked whether this initial information is sufficient, if not, then these data are taken from the corresponding tables, database or requested from the user. Selection and analysis of models (from the database models) is carried out in the required order. Then, the organization of the interaction of the selected models, calibration models (if necessary). Next, the resulting solution is analyzed. As a result, a model will be chosen to solve the problem of optimizing transport route networks, corresponding to specified conditions.

Depending on the tasks to be solved and the initial conditions (initial information), various mathematical models and methods that are presented in the model database, for example, methods based on OD-matrices, can be used in the calculations. In interactive mode, the modeling complex offers the user appropriate models for solving problems, and the user himself can specify the necessary models and methods that he will need in the calculations.

\section{Software for the system of formation and optimization of transport networks}

On the basis of the proposed mathematical apparatus, software of an automated system for the formation and optimization of route transportation networks, designed to work in a digital integrated environment, has been developed.

The automated system was developed by the staff of the department "Computing Systems, Networks and Information Security" of the Russian University of Transport. Programming language is $\mathrm{C}++$; OS are Ms. Windows $8 \mathrm{x}$ and above.

The software complies with the following basic principles necessary for functioning in the conditions of the digital integrated environment for the provision of transport: systemic unity, development, compatibility and standardization. The principle of systemic unity in the creation, operation and development of software 

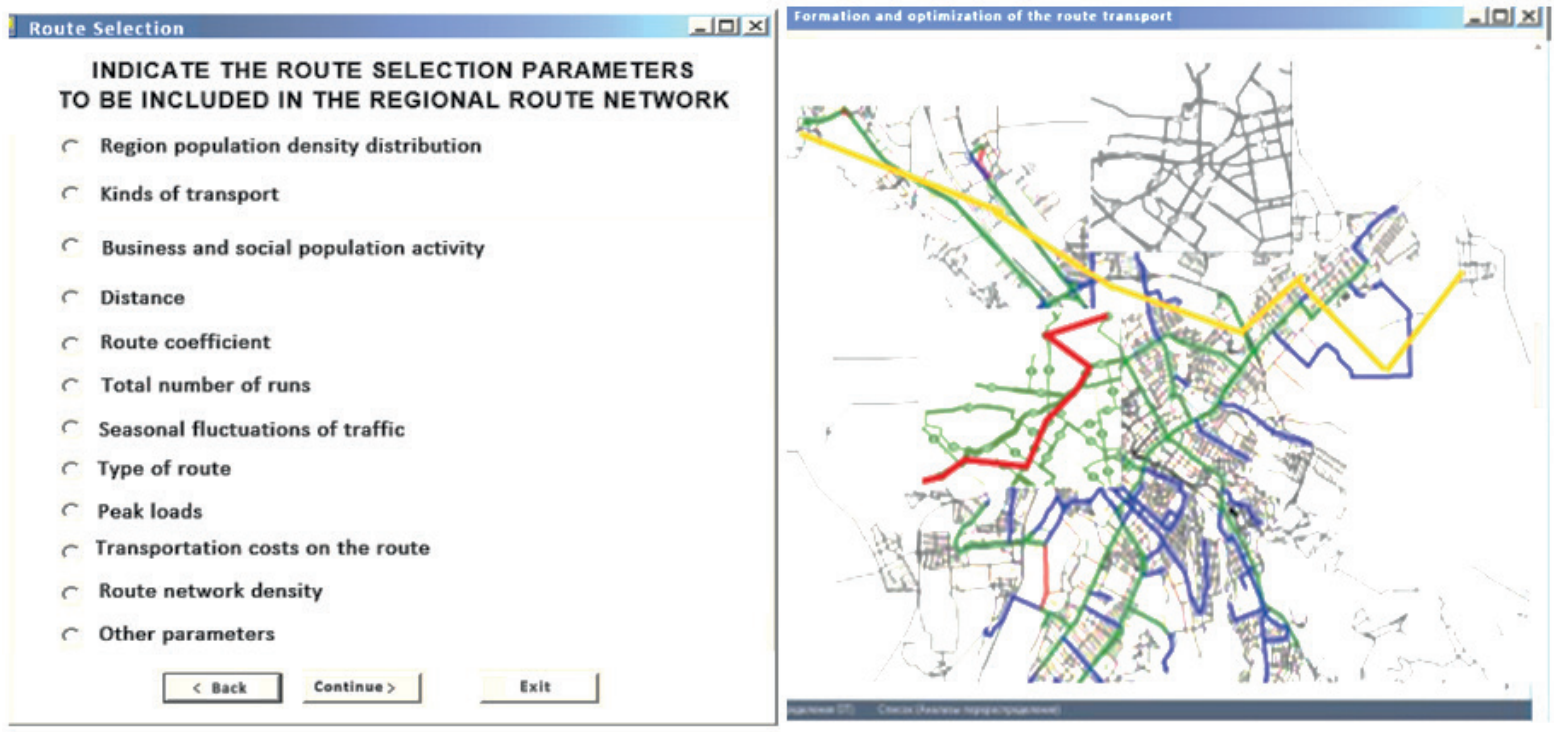

Figure 3 Software Interface

Table 1 Modelling time reduction with the developed software

\begin{tabular}{cc}
\hline Modelling Stages & Time reduction (\%) \\
\hline Information analysis & $20-30$ \\
Estimation of route parameters & $10-30$ \\
Rational options design & $10-25$ \\
Base route selection & $15-20$ \\
\hline
\end{tabular}

should ensure the integrity of the links between system components. The principle of development is that the software must be created and function, taking into account the replenishment, improvement and updating of its components. The principle of compatibility ensures the joint functioning of languages, symbols, codes, information and connections between components and preserve the open structure of the system as a whole. The principle of standardization lies in the unification, characterization and standardization of software that is invariant to routes and transport networks.

When forming the transport network in the developed system, decision-making methods are implemented both under certain and uncertain conditions of the initial information. The relations between the decision-maker and the computer goes through a series of iterative steps, with two points being significant at each iteration: the computer offers the DM a preliminary decision; the DM expresses his opinion about this decision and, if necessary, sets the direction for finding a solution. Analysis of the proposed solution is carried out in a dialogue with a computer. The route that satisfies the DM is taken as the base route.

The system interface is shown in Figure 3.

The left screen shows the selection and analysis of the parameters of the route for inclusion in the route network. The right screen shows the process of formation and optimization of the route transport network.
Table 1 shows the reduction in modelling time as a result of using the developed system.

As a result of the system operation, an electronic map with full information about the optimal (basic) route, the formed transport network, and information about the state of traffic flows will be obtained.

\section{Conclusions}

In the practical application of the developed mathematical apparatus and the developed software, the effect is expected in the following areas:

- increase the capacity of transport systems, for example, regional;

- improving the economic efficiency of the use of route rolling stock;

- attracting customers by expanding the range of services and customer-oriented approach;

- $\quad$ attracting investments from potential partners wishing to provide their services in the digital information environment;

- increasing the level of comfort and safety of route traffic.

Unlike the existing ones, the developed system allows taking into account all the parameters and characteristics of routes most fully; carry out calculations and processing of data of large dimensions; enables to solve the problems 
of formation and optimization of route transport networks more adequately and at the lowest cost, allowing to reduce the time, while ensuring efficiency of operation.

It should be noted that in subsequent articles a more detailed description of the developed mathematical apparatus will be presented, the composition and functioning of the modeling complex, including the concept of organizing the interaction of models, the algorithm for linking model parameters, the integration technique, as well as the stages of the automated system and experimental testing of the developed mathematical apparatus and System software.

\section{References}

[1] KORYAGIN, M. E. Equilibrium models of urban passenger transport system in the conditions of conflict of interests. Kuzbass State Technical University, Novosibirsk: Science, 2011. ISBN 978-5-02-032236-3.

[2] GOLDOVSKIY, Y. M., ZHELENKOV, B. V., SAFONOVA, I. E. Simulation of network database management system with limited circulation of segments. Mir Transporta Journal (MT). 2014, V.12, 4(53), p. 32-44. ISSN 1992-3252.

[3] SAFRONOV, E. A., SAFRONOV, K. E., SEMENOVA, E. S. Download management of the city transport network, given the increase in the availability of passenger transport. The Russian Automobile and Highway Industry Journal [online]. 2015, 6(46), p. 38-44 [accessed 2019-02-02]. eISSN 2658-5626. Available from: https://vestnik.sibadi.org/jour/ article/view/237?locale $=$ en_US

[4] PONOMAREVA, M. S. Coordination of carrier interests in the regional passenger traffic segment. Science studies [online]. 2015, 7(5). [accessed 2019-06-09]. ISSN 2223-5167. Available from: naukovedenie.ru/PDF/151EVN515.pdf

[5] KOLBER, Y. A. Information support of public passenger transport network optimization in major cities: relevance and approaches. The Russian Automobile and Highway Industry Journal [online]. 2018, 15(2), p. 218-228. [accessed 2019-06-09]. eISSN 2658-5626. Available from: https://doi.org/10.26518/2071-7296-2018-2-218-228

[6] SMIRNOV, M. I., KHAIROULLIN, R. Z. Mathematical models used in the optimization system of goods delivery by motor transport DISPATCHER [online]. Moscow: Preprint, Inst. Appl. Math., the Russian Academy of Science, 2002. Available from: http://www.keldysh.ru/papers/2002/prep13/prep2002 13.html

[7] SHVETSOV, V. I. Mathematical modeling of traffic flows. Automation and Remote Control. 2003, 11, p. 3-16. ISSN 0005-1179.

[8] SAFronOV, E. A., SAFronOV, K. E., SEMENOVA, E. S. Prospects of development of light rail transportation in Russian cities. The Russian Automobile and Highway Industry Journal [online]. 2016, 2(48), p. $62-70$ [accessed 201909-10]. eISSN 2658-5626. Available from: https://vestnik.sibadi.org/jour/article/view/286?locale=en_US

[9] FEDOROV, V. A. On the issue of the possibility of optimizing the route network of urban passenger transport in megalopolises. Young Scientist [online]. 2015, 2(82), p. 331-333 [accessed 2019-09-10]. eISSN 2077-8295. Available from: https://moluch.ru/archive/82/14765/

[10] CHERNORUTSKY, I. G. Decision making methods. Science, St. Petersburg: BHV-Petersburg, 2005. ISBN 5-94157-481-9.

[11] SAATY, T. L. Making decisions. Hierarchy analysis method. Translated from English by VACHNADZE, R. G. Moscow: Radio and Communication. ISBN 5-256-00443-3.

[12] GONCHAROV, V. A. Optimization methods [online]. MIET, Moscow: Jurait, 2016. ISBN 978-5-9916-3643-8. Available from: https://biblio-online.ru/bcode/378897

[13] SAFONOVA, I. E, TELNOV, G. G. The process of traffic control routing of passenger transport in the regions. In: the XIV International Scientific and Practical Conference : proceedings. Vol. 1. Innovative, Information and Communication Technologies. Moscow: Association of Alumni and Employees VVIA them. prof. Zhukovsky, 2017. ISSN 2500-1248, p. 300-302.

[14] SAFONOVA, I. E, TELNOV, G. G. Hierarchical modeling complex as a basic means of optimizing route transport networks. In: the XIV International Scientific and Practical Conference : proceedings. Vol. 1. Innovative, Information and Communication Technologies. Moscow: Association of Alumni and Employees VVIA them. prof. Zhukovsky, 2017. ISSN 2500-1248, p. 295-299.

[15] SAFONOVA, I. E, TELNOV, G. G. Selecting the optimal passenger transport routes for inclusion in the region route network. In: the XIV International Scientific and Practical Conference : proceedings. Vol. 1. Innovative, Information and Communication Technologies. Moscow: Association of Alumni and Employees VVIA them. prof. Zhukovsky, 2017. ISSN 2500-1248, p. 290-294. 